\title{
Validação do Function Observational Learning Questionnaire para o Português - Brasil
}

\author{
Taciane Adeline Castelan Hayassaka \\ Priscila Garcia Marques ${ }^{1}$ \\ Universidade Estadual de Maringá
}

\begin{abstract}
RESUMO - O objetivo deste estudo foi obter evidências de validade transcultural para a língua portuguesa - Brasil do Function of Observational Learning Questionnaire (FOLQ). Participaram do estudo atletas de natação, handebol e voleibol de ambos os sexos ( $n=362)$. O alpha de Cronbach e o coeficiente de Kappa foram utilizados para avaliar a consistência interna dos itens. Análise Fatorial Confirmatória foi realizada para verificar a adequabilidade do instrumento ao modelo conceitual. Para avaliar a fidedignidade teste-reteste, utilizou-se o teste $\mathrm{t}$ de student, $\operatorname{com} p<0,05$ para diferenças estatisticamente significativas. Os resultados mostram que foram obtidas evidências de validade para a língua portuguesa com 17 questões, mantendo os três fatores originais: habilidade, desempenho e estratégia.
\end{abstract}

Palavras-chaves: aprendizagem, habilidades motoras, psicometria, validade do teste

\section{Validation of the Function Observational Learning Questionnaire for Brazilian Portuguese}

\begin{abstract}
The aim of this study was to obtain evidence of cross-cultural validity for Brazilian Portuguese of the Function of Observational Learning Questionnaire (FOLQ). Study participants were swim, handball and volleyball athletes of both sexes $(n=362)$. Cronbach's alpha and Kappa coefficient were used to evaluate the internal consistency of the items. Confirmatory Factor Analysis was performed to verify the appropriateness of the instrument to the conceptual model. To evaluate test-retest reliability of the instrument Student's t- test was used with $p<.05$ for statistically significant differences. The results show that validity evidence was obtained for Brazilian Portuguese with 17 questions, maintaining the original three factors: skill, performance and strategy.
\end{abstract}

Keywords: learning, motor skills, psychometrics, test validity

Por tradição, aprender ou praticar uma nova habilidade motora conta com um planejamento externamente controlado pelo instrutor ou pelo professor, que decide como, quanto e qual tipo de instrução será fornecida, quantas sessões de prática o aprendiz deve realizar e qual tarefa utilizar. As habilidades motoras são adquiridas ao longo de toda a vida, pois aparecem desde a infância no engatinhar e andar sem apoio, até a aprendizagem de habilidades mais complexas para dirigir, tocar um instrumento musical ou andar de skate. A aprendizagem observacional é um dos caminhos chave pelo qual os indivíduos adquirem muitas de suas habilidades motoras (Ferrari, 1996).

Segundo Horn e Williams (2004), os estudos acerca da aprendizagem observacional iniciaram-se com a demonstração e a prática mental, dando um passo além nas contribuições sobre as possíveis explicações e intervenções na mudança de comportamentos motores e na modificação psicológica. Recentemente, a aprendizagem observacional também tem sido foco de investigação na aquisição de habilidades motoras, uma vez que, na aprendizagem motora, o uso de demonstração por modelos mais experientes, como atletas, por exemplo, é muito utilizado como facilitador para a

1 Endereço para correspondência: Campus Universitário, Av. Colombo, 5790, Jd. Universitário, Maringá, PR, Brasil. CEP: 87.020-900. E-mail: priscila.garcia.marques@gmail.com aprendizagem (Ste Marie et al., 2012). Em algumas situações, vídeos que contenham o desempenho do aprendiz podem ser utilizados como forma de fornecer feedback para dar-lhe conhecimento de resultado e para corrigir possíveis erros que estejam interferindo no desempenho de uma determinada habilidade motora (Dowrick, 2012; Paivio, 1985).

A aprendizagem observacional, assim como a aprendizagem motora de uma forma generalizada, é individualizada (Wulf, Shea, \& Lewthwaite, 2010), porque existem estratégias de instrução (Koedijker et al., 2010), artifícios de demonstração (Tani et al., 2011) e feedback (Chiviacowsky \& Wulf, 2002; 2005). Embora muito se investigue sobre os processos e mecanismos subjacentes à aprendizagem motora, cada pessoa busca motivações e metas próprias na aquisição dessas habilidades (Marques et al., 2014). Quando um aprendiz/atleta observa algo, ele procurará, de acordo com a própria meta, as variáveis de interesse a serem aprendidas.

A capacidade de aprender sozinho inspirou algumas pesquisas que consideraram variáveis de aprendizagem motora como feedback, demonstração, estabelecimento de metas e auto-observação (Huet et al., 2009; Chiwiacowsky \& Wulf, 2005; Chiwiacowsky \& Wulf, 2002; Clark \& Ste Marie, 2007; Corrêa \& Walter, 2009; Fagundes, Chen, \& Laguna, 2013; Marques, 2013, Marques et al., 2014; Schunk, 
1987; Starek \& McCullagh, 1999; Ste Marie et al., 2012). Os resultados dessas pesquisas demonstraram que, ao aprender uma habilidade motora, o aprendiz é capaz de participar ativamente no seu próprio processo de aprendizagem ao escolher suas condições de prática. Além disso, parece haver um consenso de que controlar intrinsecamente a aprendizagem a partir de referências beneficia mais o aprendiz no processo de aprendizagem do que quando se usam as formas tradicionais de aquisição de uma habilidade motora.

A aprendizagem motora intrinsecamente controlada necessita, portanto, de um padrão de referência. De acordo com Weeks (1992), a aprendizagem observacional pode melhorar a competência de um atleta para responder às constantes mudanças ambientais da sua prática esportiva. Evidências mostraram (Dowrick, 2012; Hodges \& Coppola, 2014; Ste Marie et al., 2012) que observar fotos, vídeos ou filmes de jogos ou eventos esportivos pode levar a um aumento da percepção e do desempenho nas tarefas relacionadas.

Estudos de Schunk (1987), Starek e McCullagh (1999), Law e Ste Marie (2005) e Hancock, Rymal e Ste Marie (2011) revelaram que a aprendizagem por observação provoca respostas psicológicas importantes como a motivação para mudar um comportamento, a diminuição da ansiedade e respostas cognitivas como a autoeficácia. No que se refere à aprendizagem motora, o uso da aprendizagem observacional pode mudar o comportamento do desempenho na tarefa, na habilidade e na estratégia (Cumming et al., 2005; Law \& Ste Marie, 2005).

Nas investigações sobre a aprendizagem observacional, os estudos apontaram para algumas características comuns sobre o seu uso na aquisição de habilidades motoras e/ou esportivas. A investigação de Hars e Camels (2007) sobre o uso da aprendizagem observacional em atletas de elite da ginástica artística demonstrou que os atletas usaram a aprendizagem observacional para melhorar a habilidade motora. William e Cumming (2012) investigaram a função da aprendizagem observacional e a capacidade de se imaginar em ação em atletas de elite da corrida em distância, futebol, rugby, hóquei de campo e basquete, chegando à conclusão de que, quanto mais similar for a observação à posição ocupada na modalidade esportiva, mais o uso da aprendizagem observacional volta-se para a estratégia e a habilidade.

Em virtude dos apontamentos dos estudos sobre a aprendizagem observacional, a sua função em situações práticas do cotidiano de aprendizes/atletas em contexto real passou a ser mais investigada por Cumming et al. (2005) com a elaboração de um questionário chamado Function of Observational Learning Questionnaire (FOLQ), no Canadá. Esse instrumento tem o intuito de aferir, de acordo com as características que se busca investigar - como nível competitivo, tipo de modalidade esportiva, idade e gênero - qual é a função do uso da aprendizagem observacional em uma determinada realidade, de acordo com três fatores: estratégia, desempenho e habilidade.

Esses três fatores advêm de um constructo originário de estudos derivados da Teoria Social Cognitiva de Bandura (1989) relacionados à aquisição de habilidades motoras e mais especificamente no esporte, como os estudos com a prática mental desenvolvidos por Pavio (1985). Muito do que se investiga na aprendizagem por observação considera o uso de modelos, seja de maior nível de experiência em uma determinada tarefa ou por experiência vicária (Bandura, 1989).

A estratégia está intimamente relacionada às funções executivas do córtex pré-frontal e atrelada ao estabelecimento de metas, ao planejamento e à tomada de decisão. Alinha a intenção do aprendiz/atleta de buscar formas de atuação para colocar a equipe esportiva ou ele mesmo em uma posição que se deseja alcançar (Malloy-Diniz et al., 2014). Nos esportes coletivos ou nas modalidades dependentes, cujo desempenho é fruto da interação com outros atletas, como a ginástica rítmica de grupo e o remo, por exemplo, a estratégia tem uma profunda ligação com a tática.

O desempenho é um comportamento que se observa em um determinado evento. Na aprendizagem motora, Edwards (2011) define desempenho como um comportamento motor, portanto, observável, passível de avaliação quantitativa ou qualitativa, de característica temporária, na execução de uma determinada habilidade motora. Quando se avalia a qualidade no padrão de movimento de um nadador do estilo costas em uma determinada prova ou a quantidade de chutes a gol por finalizações com sucesso de uma equipe em um jogo de futebol, é o desempenho que está sendo avaliado.

A habilidade, em específico a habilidade motora, apresenta diferentes conceituações. Tani (1988) define a habilidade motora como um movimento intencional, resultante de aprendizagem, em que o alcance de melhora na qualidade da execução é fundamentalmente importante e dependente da prática. Adams (1999) define habilidade motora como um movimento aprendido, direcionado a uma meta, realizado por uma ação neuromuscular complexamente organizada, que implica uma série de comportamentos motores. Complementar a essas definições, Guthrie (1952) aponta uma habilidade motora como um movimento intencional, que visa ao alcance de um objetivo com a máxima precisão e o mínimo gasto de energia. Em linguagem específica dos esportes de um modo geral, a habilidade motora está atrelada à técnica de movimento, embora técnica e habilidade motora não sejam sinônimos.

O instrumento FOLQ foi elaborado sob a perspectiva de tentar elencar o uso da aprendizagem observacional sob o ponto de vista da cognição e da motivação (Cumming et al., 2005). Isso significa que o pesquisador utiliza o instrumento para tentar entender os processos e mecanismos subjacentes ao funcionamento psicológico da observação de uma determinada ação. Por essa razão, o uso da aprendizagem observacional envolve três funções: estratégia, habilidade e desempenho. As funções estratégia e habilidade são aferidas e entendidas como um processo cognitivo: a estratégia preocupada com os aspectos ambientais do desempenho e a habilidade preocupada com os aspectos intrínsecos da execução de uma habilidade motora. Já a função desempenho está vinculada à aspectos motivacionais do comportamentoalvo que se deseja executar e intimamente relacionada à crença de autoeficácia (Hancock et al., 2011). 
Pesquisas têm sido realizadas buscando aferir o uso da aprendizagem observacional de acordo com a previsibilidade ambiental das modalidades esportivas (fechada - alta previsibilidade ambiental, ou aberta - pouca previsibilidade ambiental), como o estudo de Law e Hall (2009a). A aprendizagem observacional também tem sido investigada em relação ao nível competitivo dos atletas (Hancock et al., 2011; Hatami, Tahmasebi, \& Hatami Shah Mir, 2011; Sunderland, 2008; Tahmasebi, Hatami, \& Hatami Shah Mir, 2011; Wesch, Law, \& Hall, 2007), assim como em relação à idade relacionada e ao nível de experiência dos atletas (Law \& Hall, 2009b).

Em virtude da importância de viabilizar instrumentos de pesquisa que auxiliem a compreensão da aquisição de habilidades motoras e sua relação com os processos cognitivos e motivacionais para a aprendizagem observacional, este estudo tem o intuito de obter evidências de validade transcultural do FOLQ para a língua portuguesa - Brasil.

\section{Método}

\section{Participantes}

Participaram deste estudo sete profissionais (quatro tradutores e três peritos), os quais foram envolvidos nos processos de tradução, adaptação e validação de conteúdo da versão na língua portuguesa do FOLQ, e 362 atletas de ambos os sexos do estado do Paraná, de nível competitivo nacional e internacional. Esse nível de experiência aumenta a confiabilidade em refletir sua relação com as características de previsibilidade ambiental da modalidade esportiva. Optou-se por uma modalidade individual - natação - e duas modalidades esportivas coletivas, com características diferentes, uma de invasão - handebol - e outra de não invasão - voleibol (Reverdito \& Scaglia, 2009). A previsibilidade ambiental no desempenho das habilidades motoras também foi considerada (Edwards, 2011): o handebol, classificado como habilidade motora aberta ou baixa previsibilidade ambiental; o voleibol, considerado como de habilidade motora com ambiente de prática semiprevisível, e a natação, classificada como habilidade motora fechada (alta previsibilidade ambiental), para que fosse possível abranger diferentes características das modalidades esportivas no âmbito de sua prática. $\mathrm{O}$ estudo teve aprovação do comitê de ética local.

\section{Instrumento}

O instrumento original utilizado foi o FOLQ, elaborado por Cumming et al. (2005), que tem a função de avaliar o que os atletas aprendem por observação para melhorar o próprio desempenho esportivo. O questionário é composto por 17 itens. Cada item se refere a uma dimensão da aprendizagem observacional em atletas (Cumming et al., 2005): habilidade, estratégia e desempenho. As respostas são dadas em uma escala do tipo Likert de sete pontos, que variam de raramente
(1) a frequentemente (7). Os resultados são atribuídos a cada subescala, obtidos a partir da média aritmética das respostas dadas aos itens correspondentes a cada dimensão da aprendizagem observacional.

\section{Procedimentos}

A primeira etapa consistiu da obtenção da autorização formal para tradução e uso desse instrumento no Brasil, junto à autora do instrumento. Dois tradutores (de língua materna portuguesa) fizeram a tradução do FOLQ para o português. Cada um deles fez o trabalho de tradução de forma independente, sem o conhecimento de que outro profissional estava engajado a fazer o mesmo trabalho. Em um segundo momento, outros dois tradutores profissionais retraduziram para o inglês as versões traduzidas para o português, de forma independente.

Em seguida, realizou-se a avaliação e a modificação das versões preliminares. As duas traduções para a língua inglesa foram comparadas com a versão original do instrumento, e a semântica assim como os termos técnicos necessários no instrumento foram discutidos e adequados à compreensão dos aprendizes (Pasquali, 2010; Vallerand, 1989).

Professores peritos na área de Aprendizagem Motora foram convidados a participar, no papel de juízes avaliadores, para verificar a adequação dos itens do FOLQ quanto à pertinência prática, clareza de linguagem e dimensão teórica dos itens. A avaliação nessa etapa consistiu em categorizar a adequação dos itens de 0 (inadequado) a 5 (adequado).

\section{Análise Teórica dos Itens}

Após as etapas descritas acima, o cálculo do Coeficiente de Validade do Conteúdo (CVC) para a clareza da linguagem e para a pertinência prática foi conduzido (Hair et al., 2009), adotando-se um CVC superior a 0,80 para garantir o rigor, indicando índices altamente aceitáveis de validade de conteúdo. Para a avaliação da dimensão teórica, foi calculado o índice de concordância entre avaliadores, o coeficiente Kappa (Pasquali, 2010), com coeficiente acima de 0,80 considerado adequado. Após esses cálculos, o instrumento piloto foi elaborado para fins de coleta de dados.

\section{Procedimentos para a Coleta de Dados do Instrumento Piloto}

A aplicação da versão piloto do questionário foi realizada nos locais de treinamento dos atletas, em pequenos grupos antes dos treinamentos. As instruções de como responder ao instrumento foram apresentadas pelas pesquisadoras do estudo. Cada atleta respondeu ao instrumento individualmente. $\mathrm{O}$ teste e o reteste foram conduzidos para verificar a fidedignidade do instrumento. $\mathrm{O}$ reteste se deu 14 dias após a aplicação do teste. 


\section{Análise dos Dados}

Os dados obtidos foram avaliados com o auxílio do software SPSS (versão 15.0 para Windows). Em um primeiro momento, foi realizada a análise de cluster para agrupamento das questões de acordo com os fatores: estratégia, habilidade e desempenho. Os dados dos respondentes foram divididos em duas amostras ( $n=181$ cada). A análise discriminante foi conduzida no primeiro momento para um perfilhamento dos dados, em virtude de que os fatores do questionário já são definidos em habilidade, desempenho e estratégia. A distância de Mahalanobis foi calculada para identificar os outliers, considerando o critério de $p<0,001$ e a maior distância $\left(\mathrm{D}^{2}\right)$ encontrada. Como resultado, 51 casos foram eliminados. Com a análise dos dados faltantes, mais 27 casos foram eliminados, ficando a amostra com 284 casos, que foram novamente reagrupados para a análise confirmatória.

O coeficiente de Kappa foi utilizado para verificar a consistência interna entre os três fatores: desempenho, habilidade e estratégia. O coeficiente do alpha de cronbach foi utilizado para a análise da consistência interna das respostas da amostra intra fatores.

A Análise Fatorial Confirmatória foi realizada para verificar o ajuste do modelo quanto à validade de constructo considerando a unidimensionalidade, porque se presumiu que nenhum dos itens medidos é determinado por mais de um fator (nesse caso, habilidade, desempenho e estratégia). Para a avaliação da validade do modelo, é necessária a identificação de quatro medidas: (a) estimativa dos caminhos; (b) resíduos padronizados; (c) índices de modificação; e (d) busca de identificação. Para identificar essas medidas, utilizou-se a função qui-quadrado de ajuste mínimo $\left(\chi^{2}\right)$, os graus de liberdade (df), a raiz padronizada do resíduo médio (SRMR), a raiz do erro quadrático médio de aproximação (RMSEA), o índice de ajuste comparativo (CFI) e o índice de Tucker-Lewis (TLI). Todas as medidas encontradas dão informação sobre a qualidade de ajuste do modelo para a validação do questionário (Hair et al., 2009). A análise fatorial confirmatória foi conduzida pelo software AMOS 20.0. O teste e reteste para verificação da fidedignidade da versão final do questionário foi verificada pelo teste $\mathrm{t}$ de student pareado. A comparação entre os fatores habilidade, desempenho e estratégia foi feita pelo teste $t$ de student para amostras dependentes. Adotou-se $p<0,05$ para diferenças estatisticamente significativas.

\section{Resultados}

A consistência interna para cada subescala do FOLQ Brasil foi calculada usando o alpha de Cronbach. O nível de critério estabelecido para a escala foi o coeficiente de 0,70, de acordo com Nunnally e Berstein (1994). Para a subescala habilidade, o coeficiente encontrado foi de 0,831 ; para o desempenho foi de 0,775; e, para a estratégia, foi de 0,82 . Houve uma correlação moderada, porém significativa, entre as três subescalas, com índices entre 0,40 até 0,58 em magnitude, com $\mathrm{p}<0,01$ e com coeficiente de Kappa de
0,071 para a relação estratégia e desempenho, 0,083 para a relação habilidade e desempenho, e 0,076 para a relação estratégia e habilidade.

O resultado da análise fatorial confirmatória se encontra na Tabela 1. Todos os dados do modelo foram testados utilizando o $\chi^{2}$. De acordo com Hair et al. (2009), um valor de $\chi^{2}$ amplo indica um modelo pouco ajustado para a análise confirmatória, ao passo que um valor menor de $\chi^{2}$ indica um bom modelo. Como é possível observar na Tabela 2, o valor de $\chi^{2}$ foi reduzido, indicando um bom ajuste do modelo para a proposta deste estudo, com significância estatística encontrada em $\mathrm{p}<0,01$. O SRMR foi utilizado porque é uma medida absoluta calculada a partir da diferença entre a variância e a covariância da amostra e a estimativa da variância e covariância da população (Tabachnick \& Fidell, 2001). Um valor de SRMR menor que 0,08 indica um bom modelo. Para o modelo do presente estudo, o SRMR foi de 0,05. O CFI também testa em comparação o quão melhor é o ajustamento do modelo para um modelo independente, a partir do cálculo do TLI. De acordo com Hair et al. (2009), valores próximos de 0,95 para ambos (CFI e TLI) indicam

Tabela 1. Índice de qualidade do ajuste do Function of Observational Learning Questionnaire - Brasil

\begin{tabular}{ccccccc}
\hline Amostra & $\chi^{2}$ & Df & SRMR & RMSEA & CFI & TLI \\
\hline $\mathrm{n}=282$ & 84.28 & 41 & 0,05 & 0,07 & 0,94 & 0,92 \\
\hline
\end{tabular}

Tabela 2. Cargas fatoriais padronizadas e erro dos itens das subescalas do Function of Observational Learning Questionnaire - Brasil (n=282)

\begin{tabular}{ccc}
\hline Item & Cargas Fatoriais & Erro \\
\hline Habilidade & 0,75 & 0,51 \\
01 & 0,71 & 0,64 \\
07 & 0,72 & 0,59 \\
09 & 0,78 & 0,61 \\
12 & 0,87 & 0,65 \\
13 & 0,70 & 0,47 \\
17 & & \\
Estratégia & 0,77 & 0,59 \\
02 & 0,73 & 0,62 \\
03 & 0,86 & 0,68 \\
04 & 0,72 & 0,53 \\
10 & 0,71 & 0,59 \\
14 & & \\
Desempenho & 0,76 & 0,54 \\
05 & 0,70 & 0,49 \\
06 & 0,76 & 0,61 \\
08 & 0,66 & 0,59 \\
11 & 0,74 & 0,63 \\
15 & 0,72 & 0,47 \\
16 & & \\
\hline
\end{tabular}

Nota: Número do item corresponde ao número da questão no FOLQ Brasil 
um bom modelo. Para este estudo, o CFI encontrado foi de 0,94 e o TLI foi de 0,92. Por fim, o RMSEA mostra como a aproximação dos dados do modelo testado se dá da matriz de covariância, expressa como a discrepância pelos graus de liberdade. Para o presente estudo, o valor para o RMSEA demonstra um bom ajustamento do modelo para essa variável $(\leq 0,07)$. O valor do fator padronizado para cada item do FOLQ - Brasil mostra que cada item contribui para a subescala na qual está contido, porque os valores não excedem 1,00, considerado o limite aceitável para a alocação dos itens em suas subescalas. A Tabela 2 mostra esses valores.

Dados os resultados encontrados, o FOLQ - Brasil obteve evidências de validade mantendo as 17 questões do questionário de origem. A Tabela 3 apresenta essas questões, com a referida média e desvio padrão para cada uma das questões, no teste e reteste. Não foram encontradas diferenças estatisticamente significativas, o que demonstra boa estabilidade temporal da versão final do instrumento

O FOLQ - Brasil manteve os três fatores originais: desempenho, habilidade e estratégia. Seguindo a Tabela 3, as afirmações contidas no fator desempenho são 3, 6, 8, 11, 14 e 16; para o fator habilidade, as questões contidas são 1, 7, $9,12,13$ e 17; e, por fim, para o fator estratégia, as questões são $2,4,5,10$ e 15 . O fator de maior uso para a aprendizagem observacional pelo atleta será o que obtiver maior soma aritmética nos valores atribuídos pela escala Likert para as questões contidas dentro do fator correspondente.

Quanto aos resultados encontrados no questionário, para a natação, a média encontrada para os fatores foi: habilidade: $5,72 \pm 1,34, \mathrm{p}<0,01$; desempenho: 4,11 $\pm 1,74, \mathrm{p}<0,00$; e estratégia: $4,12 \pm 1,21, p<0,01$. Para o handebol, a média encontrada para os fatores foi: habilidade: $5,53 \pm 1,52, \mathrm{p}<$
0,05; desempenho: 4,20 $\pm 2,06, \mathrm{p}<0,00$; e estratégia: 5,03 $\pm 1,51, \mathrm{p}<0,01$. Para o voleibol, a média encontrada para os fatores foi: habilidade: $5,56 \pm 1,12, p<0,05$; desempenho: $4,54 \pm 1,71, \mathrm{p}<0,01$; e estratégia: $5,23 \pm 1,98, \mathrm{p}<0,01$.

\section{Discussão}

A proposta do presente estudo foi a validação transcultural do FOLQ - Brasil, originalmente elaborado por Cumming et al. (2005). Em princípio, o FOLQ foi elaborado a partir da similaridade entre aprendizagem observacional e prática mental (Cumming et al., 2005; Paivio, 1985). De acordo com Paivio (1985), a aquisição de habilidades esportivas é regulada por aspectos cognitivos e motivacionais. Assim, as pessoas que se interessam por determinada habilidade motora ou modalidade esportiva buscam observar aquilo que as impulsiona, seja para uso cognitivo (melhorar a tática de jogo ou criar uma jogada nova, por exemplo) ou por influência de motivação, porque a jogada foi bonita, por exemplo. $\mathrm{O}$ papel da aprendizagem observacional está consolidado em importância e influência sobre a aquisição de habilidades motoras e/ou esportivas (Ferrari, 1996).

O FOLQ (Cumming et al. 2005) foi construído e validado com três fatores atrelados ao uso da aprendizagem observacional: habilidade, desempenho e estratégia. $\mathrm{Na}$ literatura sobre demonstração, que é a forma de aprendizagem observacional mais investigada no Brasil, as pesquisas apontam que os aprendizes observadores podem adquirir informações importantes sobre feedback relacionado aos resultados (conhecimento de resultado) e sobre o padrão de movimento (conhecimento de performance), além de

Tabela 3. Itens do FOLQ - Brasil ( $n=282)$ no teste e reteste.

\begin{tabular}{|c|c|c|c|c|}
\hline & \multirow{2}{*}{ Itens } & \multicolumn{3}{|c|}{ Reteste } \\
\hline & & $M(d p)$ & $M(d p)$ & $p$ \\
\hline 1. & Eu uso AO para mudar a execução de uma habilidade. & $5,84(1,18)$ & $5,72(1,12)$ & 0,12 \\
\hline 2. & Eu uso AO para determinar como uma estratégia será empregada em um evento/jogo. & $5,38(1,51)$ & $5,18(1,43)$ & 0,08 \\
\hline 3. & Eu uso AO para entender quais detalhes são importantes. & $5,72(1,34)$ & $5,61(1,27)$ & 0,06 \\
\hline 4. & Eu uso AO para formar planos alternativos. & $5,22(1,54)$ & $4,89(1,65)$ & 0,06 \\
\hline 5. & Eu uso AO para fazer novos planos e estratégias na minha mente. & $5,06(1,38)$ & $5,29(1,30)$ & 0,13 \\
\hline 6. & Eu uso AO para responder melhor à excitação associada com o desempenho. & $4,78(1,46)$ & $4,41(1,39)$ & 0,06 \\
\hline 7. & Eu uso AO para me ajudar aperfeiçoar minhas habilidades. & $5,99(1,13)$ & $6,01(1,24)$ & 0,08 \\
\hline 8. & Eu uso AO para aprender como manter o foco durante situações inesperadas. & $5,08(1,55)$ & $4,87(1,72)$ & 0,08 \\
\hline 9. & Eu uso AO para entender como aperfeiçoar a execução de uma habilidade. & $5,72(1,20)$ & $5,69(1,08)$ & 0,06 \\
\hline 10. & Eu uso AO para me ajudar melhorar minhas estratégias no evento/jogo. & $5,43(1,28)$ & $5,31(1,03)$ & 0,07 \\
\hline 11. & Eu uso AO para aprender como lidar com a ansiedade. & $3,90(1,77)$ & $4,16(1,81)$ & 0,34 \\
\hline 12. & Eu uso AO para me ajudar a aprender novas habilidades. & $5,67(1,33)$ & $4,98(1,25)$ & 0,07 \\
\hline 13. & Eu uso AO para melhorar minhas habilidades & $5,24(1,37)$ & $5,03(1,27)$ & 0,08 \\
\hline 14. & Eu uso AO para entender como me manter motivado. & $4,95(1,60)$ & $5,12(1,55)$ & 0,21 \\
\hline 15. & Eu uso AO para desenvolver planos e rotinas na minha prática esportiva. & $4,97(1,56)$ & $5,01(1,43)$ & 0,08 \\
\hline 16. & Eu uso AO para me manter otimista nas situações dolorosas (ex. uma torção no tornozelo, etc.). & $4,15(1,87)$ & $4,79(1,66)$ & 0,06 \\
\hline 17. & Eu uso AO para me ajudar a executar adequadamente uma habilidade física. & $5,59(1,35)$ & $5,39(1,24)$ & 0,07 \\
\hline
\end{tabular}

Nota: Os participantes responderam em uma escala likert de 1(raramente) a 7 (frequentemente). Legenda: AO - Aprendizagem Observacional 
receber informações valiosas sobre o movimento a ainda ser desempenhado, vindos de outros modelos mais experientes (Marques, 2013; Ste Marie et al., 2012; Tani et al., 2011). Estudos têm mostrado que a observação de modelos pode levar à melhora na forma do movimento (Fagundes, Chen, \& Laguna, 2013) e na execução da habilidade relacionada ao aumento da autoeficácia (Law \& Ste Marie, 2005), demonstrando ainda relações com o foco de atenção na tarefa a ser executada (Horn, Williams, \& Scott, 2002) e a correção de erros (Dowrick, 2012). No entanto, pouco a pouco, os estudos tendem a investigar de forma mais prática, fora de ambiente laboratorial, quais as funções exercidas pela aprendizagem observacional no contexto da prática esportiva, como mostra o estudo de Marques (2013). Para a obtenção de um instrumento que pudesse focar mais especificamente essas funções, buscaram-se evidências da validade transcultural do FOLQ- Brasil, para se ter um recurso a mais para pesquisas.

Os resultados de Law e Hall (2009a) mostraram que atletas de modalidades dependentes (cujo desempenho é fruto da interação com outro atleta), de habilidades motoras semiabertas ou abertas (baixa previsibilidade ambiental), tiram proveito da aprendizagem observacional para melhorar a habilidade, o que reforça o resultado encontrado pelo presente estudo, visto que, para as modalidades voleibol e handebol, o fator habilidade foi o mais apontado pelos atletas.

Por outro lado, o estudo de Law e Hall (2009a) demonstrou que atletas de modalidades independentes, de alta previsibilidade ambiental, no caso a corrida, frequentemente apontaram o fator desempenho como o mais importante, diferente do resultado encontrado neste estudo, em que os atletas de natação apontaram o fator habilidade como maior função da aprendizagem observacional. Essa diferença provavelmente ocorreu porque a natação é uma modalidade em que braços, pernas, tronco e movimentos respiratórios devem ser complexamente coordenados no ambiente aquático para um bom desempenho esportivo. Ao observar outros atletas nadando, o observador busca extrair dicas mecânicas para aprimorar o nado.

Os resultados do presente estudo corroboram ainda os resultados encontrados por Sunderland (2008) e Wesch, Law e Hall (2007), que apontaram a habilidade como sendo a função da aprendizagem observacional mais utilizada por atletas em nível competitivo.

De acordo com Cumming et al. (2005), o uso da aprendizagem observacional pode modificar estruturas psicológicas do atleta, sendo que, no que tange ao contexto esportivo, a cultura assim como o país de origem têm uma influência importante nas motivações que levam os atletas a se engajarem nas atividades ou inclusive abandoná-las (Rocha \& Santos, 2010). Assim, é importante que a área de Aprendizagem Motora assim como a Psicologia do Esporte tenham um instrumento válido para a nossa cultura que também nos permita investigar a função da aprendizagem observacional relacionada às modalidades esportivas e outras variáveis intervenientes.

Como demonstrado neste estudo, o FOLQ - Brasil atendeu aos procedimentos metodológicos para ser validado para a população brasileira (Hair et al., 2009; Tabachnick \& Fidell, 2001; Vallerand, 1989). No estudo original de exploração e confirmação do questionário na língua inglesa, a validade concorrente e a validade de constructo foram rigorosamente atendidas, assim como os pressupostos de fidedignidade. $\mathrm{O}$ presente estudo também atendeu rigorosamente a validade de constructo. Assim, o questionário atende aos três fatores originais: desempenho, habilidade e estratégia.

Há numerosas possibilidades de uso do FOLQ em futuros estudos no Brasil. Por exemplo, há a possibilidade de se investigar a aprendizagem observacional de acordo com o nível competitivo e o tipo de modalidade esportiva, diferenças entre atletas novatos e experientes, o uso da aprendizagem observacional por atletas e técnicos de diferentes níveis competitivos e, ainda, a relação da aprendizagem observacional com outras variáveis psicológicas e motoras intervenientes à prática esportiva.

Em conclusão, o FOLQ validado para a língua portuguesa manteve suas características psicométricas da versão original. No entanto, futuras pesquisas podem ajudar na compreensão do quanto essas funções são representativas quanto aos resultados na prática esportiva e a sua relação com as metas estabelecidas para o que se espera com o esporte por parte dos atletas e também dos técnicos. Com isso, assim como já se discute na teoria social cognitiva de Bandura (1989), espera-se que a aprendizagem observacional ocupe um lugar de importante relevância na área da Aprendizagem Motora e da Psicologia do Esporte.

\section{Referências}

Adams, D. L. (1999). Develop better motor skill progressions with Gentile's Taxonomy of tasks. Journal of Physical Education, Recreation and Dance, 70(8), 35-38.

Bandura, A. (1989). Social cognitive theory. In R. Valta (Ed.), Annals of child development, 6 (pp.1-60). Greenwich: JAI Press.

Chiviacowsky, S., \& Wulf, G. (2002). Self-controlled feedback: Does it enhance learning because performers get feedback when they need it? Research Quarterly for Exercise and Sport, 73, 408-415. doi:10.1080/02701367.2002.10609040

Chiviacowsky, S., \& Wulf, G. (2005). Self-controlled feedback is effective if it is based on the learner's performance. Research Quarterly for Exercise and Sport, 76, 42-48. doi:10.1080/027 01367.2005.10599260

Clark, S. E., \& Ste Marie, D. M. (2007). The impact of self-as-amodel interventions on children's self-regulation of learning and swimming performance. Journal of Sport Science, 25, 577-586.

Corrêa, U. C., \& Walter, C. (2009). A autoaprendizagem motora: Um olhar para alguns dos fatores que afetam a aquisição de habilidades motoras. In M. T. Cattuzzo \& G. Tani (Eds.), Leituras em biodinâmica e comportamento motor: Conceitos e aplicações (pp. 231-261). Recife: Editora da Universidade de Pernambuco.

Cumming, J., Clark, S. E., Ste-Marie, D. M., McCullagh, P., \& Hall, C. (2005). The functions of observational learning questionnaire (FOLQ). Psychology of Sport and Exercise, 6, 517-537.

Dowrick, P. W. (2012). Self-modeling: Expanding the theories of learning. Psychology in the Schools, 49(1), 30-41. doi: $10.1002 /$ pits. 20613 
Edwards, W. H. (2011). Motor learning and control: From theory to practice. Balmont: Wadsworth Cengage Learning.

Fagundes, J., Chen, D. D., \& Laguna, P. (2013). Self-control and frequency of model presentation: Effects on learning a ballet passé releve. Human Movement Science, 32, 847-856.

Ferrari, M. (1996). Observing the observer: Self-regulation in the observational learning of motor skills. Developmental Review, 16, 203-40.

Guthrie, E. R. (1952). The psychology of learning. New York: Harper \& Row.

Hair, J. F., Black, W. C., Babin, B. J., Anderson, R. E., \& Tatham, R. L. (2009). Análise multivariada dos dados (A. S. Sant'Ana, Trans., $6^{\mathrm{a}}$ ed.). Porto Alegre: Bookman.

Hall, C. R., Munroe-Chandler, K. J., Cumming, J., Law, B., Ramsey, R., \& Murphy, L. (2009). Imagery and observational learning use and their relationship to sport confidence. Journal of Sports Sciences, 24(4), 327-337. doi: 10.1080/02640410802549769.

Hancock, D. J., Rymal, A.M., \& Ste Marie, D. M. (2011). A triadic comparison of the use the observational learning amongst athletes, coaches, and officials. Psychology of Sport and Exercise, 12(3), 236-241. doi: 10.1016/j. psychsport.2010.11.002

Hars, M., \& Calmels, C. (2007). Observation of elite gymnastic performance: Processes and perceived functions of observation. Psychology of Sport and Exercise, 8(3), 337-354. doi: 10.1016/j.psychsport.2006.06.004

Hatami, F., Tahmasebi, F., \& Hatami Shah Mir, E. (2011) The effects of age, gender and sport type on observational learning use in elite athletes. Motor Behavior (Research on Sport Science), 3(8), 35-48.

Horn, R. R., \& Williams, A. M. (2004). Observational learning: Is the time we look another look? In A. M. Williams \& N. J. Hodges (Eds.), Skill Acquisition in Sport: Research, theory and Practice (pp. 175-206). London: Routledge.

Horn, R. R.,Williams, A. M., \& Scott, M. A. (2002). Learning from demonstrations: The role of visual search during observational learning from video and point-light models. Journal of Sports Sciences, 20(3), 253-269. doi: 10.1080/026404102317284808

Hodges, N. J., \& Coppola, T. (2014) What we think we learn from watching others: The moderating role of ability on perceptions of learning from observation. Psychological Research, 79(4), 609-620. doi: 10.1007/s00426-014-0588-y

Huet, M., Camachon, C., Fernandez, L., Jacobs, D. M., \& Montagne, G. (2009). Self-controlled concurrent feedback and the education of attention towards perceptual invariants. Human Movement Science, 28(4), 450-467. doi: dx.doi.org/10.1016/j. humov.2008.12.004.

Koedijker, J. M., Poolton, J. M., Maxwell, J. P., Oudejans, R. R. D., Beek, P. J., \& Masters, R. S. W. (2011). Attention and time constraints in perceptual-motor learning and performance: Instruction, analogy, and skill level. Consciousness and Cognition, 20(2), 245-256. doi: http://dx.doi.org/10.1016/j. concog.2010.08.002.

Law, B., \& Ste-Marie, D. M. (2005). Effects of self-modeling on figure skating jump performance and psychological variables. European Journal of Sport Science, 5, 143-152.

Law, B., \& Hall, C. (2009a) Observation Learning use and selfefficacy beliefs in adult sport novices. Psychology of Sport and Exercise, 10, 263-270.
Law, B., \& Hall, C. (2009b). The relationships among skill level, age, and golfers' observational learning use. The Sport Psychologist, 23, 42-58.

Marques, P. G. (2013). O efeito do controle do aprendiz sobre a auto-observação na aprendizagem motora (Unpublished doctoral dissertation). Escola de Educação Física e Esportes da Universidade de São Paulo, São Paulo.

Marques, P.G., Walter, C., Tani, G., \& Corrêa, U. C. (2014). O efeito do auto estabelecimento de metas na aquisição de uma habilidade motora. Revista Motricidade, 10(4), 56-63. doi: http://dx.doi.org/10.6063/motricidade.10(4).2981

Malloy-Diniz, L.F., Jardim de Paula, J., Sedó, M., Fuentes, D., \& Leite, W.B. (2014). Neuropsicologia das funções executivas e da atenção. In D. Fuentes, L. F. Malloy-Diniz C. H. P. Camargo, \& R. M. Cosenza (Orgs.) Neuropsicologia: Teoria e Prática (pp. 115-138). Porto Alegre: Artmed.

Nunnally, J. C., \& Berstein, I. H. (1994). Psychometric theory (3rd ed.). New York: McGraw Hill.

Pasquali, L. (2010). Instrumentação psicológica: Fundamentos e prática. Porto Alegre: Artmed.

Paivio, A. (1985). Cognitive and motivational functions of imagery in human performance. Canadian Journal of Applied Sports Sciences, 10, 22S-28S.

Reverdito, R. S., \& Scaglia, A. C. (2009). Pedagogia do Esporte: Jogos coletivos de invasão. São Paulo: Phorte.

Rocha, P. G. M., \& Santos, E. S. (2010). O abandono da modalidade esportiva na transição da categoria juvenil e para adulto: Estudo com talentos do atletismo. Revista de Educação Física/UEM, 21(1), 60-77. doi: 10.4025/reveducfis.v21i1.6912.

Schunk, D. (1987). Peer models and children's behavioral change. Review of Educational Research, 57, 149-174.

Starek, J., \& McCullagh, P. (1999). The effect of self-modeling on the performance of beginning swimmers. The Sport Psychologist, 13(3), 269-287.

Ste-Marie, D. M., Law, B., Rymal, A.M., Jenny, O., Hall, C., \& McCullagh, P. (2012). Observational performance for motor skill learning and performance: An applied model for the use of observation. International Review of Sport and Exercise Psychology, 5(2), 145-176.

Sunderland, A. J. (2008). The effects of moderating variables on the functions of observational learning (Unpublished doctor al dissertation). University of Ottawa, Ottawa, Ontario, Canada.

Tahmasebi, F., Hatami, F., \& Hatami Shah Mir, E. (2011) The relationships between the functions of observational learning and self-confidence in elite athletes. Sport Sciences Quarterly, 2(6), 113-131.

Tani, G., Bruzi, A. T., Bastos, F. H., \& Chiviacowsky, S. (2011). $\mathrm{O}$ estudo da demonstração em aprendizagem motora: Estado da arte, desafios e perspectivas. Revista Brasileira de Cineantropometria e Desenvolvimento Humano, 13(5), 392403. doi: 10.5007/1980-0037.2011v13n5p392.

Tabachnick, B. G., \& Fidell, L.S. (2001). Using multivariate statistics (4th ed.). New York, NY: Harper Collins.

Vallerand, R. J. (1989). Vers une méthodologie de validation transculturelle des questionnaries psychologiques: Implications pour la recherche em lanque française. Psychologie Canadiense, 30, 662-680.

Weeks, D. L. (1992). A comparision of modeling modalities in the observational learning of na externally paced task. Research Quarterly for Exercise and Sport, 63, 373-380. 
Wesch, N. N, Law, B., \& Hall, C. R. (2007). The Use of Observational Learning by Athletes. Journal of Sport Behavior; 30(2), 219-231.

Williams, S. E., \& Cumming, J. (2012). Athletes' ease of imaging predicts their imagery and observational learning use. Psychology of Sport and Exercise, 13(4), 363-370.
Wulf, G., Shea, C., \& Lewthwaite, R. (2010), Motor skill learning and performance: A review of influential factors. Medical Education, 44, p.75-84. doi: 10.1111/j.13652923.2009.03421.x

Recebido em 22.08.2014

Primeira decisão editorial em 02.11.2015

Versão final em 16.11.2015

Aceito em 10.02.2016 